\title{
A Note on the McKelvey Uncovered Set and Pareto Optimality
}

\author{
Felix Brandt • Christian Geist • Paul \\ Harrenstein
}

Received: date / Accepted: date

\begin{abstract}
We consider the notion of Pareto optimality under the assumption that only the pairwise majority relation is known and show that the set of necessarily Pareto optimal alternatives coincides with the McKelvey uncovered set. As a consequence, the McKelvey uncovered set constitutes the coarsest Pareto optimal majoritarian social choice function. Moreover, every majority relation is induced by a preference profile in which the uncovered alternatives precisely coincide with the Pareto optimal ones. We furthermore discuss the structure of the McKelvey covering relation and the McKelvey uncovered set.
\end{abstract}

Keywords Pareto optimality $\cdot$ McKelvey uncovered set $\cdot$ majoritarian social choice

This material is based on work supported by Deutsche Forschungsgemeinschaft under grants BR 2312/7-2 and BR 2312/9-1. Paul Harrenstein is supported by the ERC under Advanced Grant 291528 ("RACE"). The paper has benefitted from discussions at the 12th Meeting of the Society for Social Choice and Welfare in Boston (June, 2014) as well as the Dagstuhl Seminar on Computational Social Choice (June, 2015). The authors furthermore thank Jean-François Laslier and Hans Georg Seedig for helpful discussions and technical support.

F. Brandt

Technische Universität München

Munich, Germany

E-mail: brandtf@in.tum.de

C. Geist

Technische Universität München

Munich, Germany

E-mail: geist@in.tum.de

P. Harrenstein

University of Oxford

Oxford, United Kingdom

E-mail: paul.harrenstein@cs.ox.ac.uk 


\section{Introduction}

Let $A$ be a finite set of $m$ alternatives. The preferences of an agent $i$ over these alternatives are represented by a complete, transitive, and antisymmetric preference relation $R_{i} \subseteq A \times A{ }^{1}$ The interpretation of $(x, y) \in R_{i}$, usually denoted by $x R_{i} y$, is that agent $i$ values alternative $x$ at least as much as alternative $y$. In accordance with conventional notation, we write $P_{i}$ for the strict part of $R_{i}$, i.e., $x P_{i} y$ if $x R_{i} y$ but not $y R_{i} x$. Since $R_{i}$ is antisymmetric, $x P_{i} y$ iff $x R_{i} y$ and $x \neq y$. A preference profile $R$ is a finite vector of pairs which associate each agent $i$ with its corresponding preference relation $R_{i}$. For a given preference profile, $N_{R}$ denotes the set of agents represented in $R$. In particular, we do not assume a fixed number of agents. For convenience, we furthermore define $n_{R}(x, y):=\left|\left\{i \in N_{R} \mid x R_{i} y\right\}\right|$. The relations $R_{\text {Maj }}$ and $R_{\text {Par }}$ of a given preference profile $R$ are then given by

$$
\begin{aligned}
& x R_{\text {Maj }} y \text { iff } n_{R}(x, y) \geq n_{R}(y, x), \text { and } \\
& x R_{\text {Par }} y \text { iff } n_{R}(x, y)=\left|N_{R}\right|
\end{aligned}
$$

By convention, again $P_{\mathbf{M a j}}$ denotes the strict part of $R_{\mathbf{M a j}}$ and $P_{\mathbf{P a r}}$ the strict part of $R_{\mathbf{P a r}}$. Thus, $x P_{\mathbf{P a r}} y$ iff $x R_{i} y$ for all $i$ and $x P_{j} y$ for some $j$. The relation $R_{\text {Maj }}$ will be called the majority relation and $P_{\mathbf{P a r}}$ the Pareto relation of $R$, respectively. Note that, by definition, $R_{\mathrm{Maj}}$ is complete whereas the Pareto relation $P_{\mathbf{P a r}}$ is transitive and antisymmetric.

We say that a profile $R$ is consistent with the majority relation $R_{\mathrm{Maj}}^{\prime}$ of another profile $R^{\prime}$ if $R_{\mathrm{Maj}}=R_{\mathrm{Maj}}^{\prime}$. In this case, $R$ is also said to be consistent with $R^{\prime}$

An alternative $x \in A$ is called a Condorcet winner of a given (majority) relation $R_{\mathrm{Maj}}$ if it strictly dominates all other alternatives, i.e., if $x P_{\mathbf{M a j}} y$ for all $y \in A \backslash\{x\}$.

Given a preference profile $R$, an alternative $x$ is said to be Pareto optimal whenever there is no alternative $y$ with $y P_{\mathbf{P a r}} x$, i.e., if $x$ is a maximal element of $R_{\mathrm{Par}}{ }^{2}$

A social choice function (SCF) $f$ associates with every preference profile $R$ over a set $A$ of alternatives a nonempty subset $f(R)$ of $A$. An SCF $f$ is called a refinement of another SCF $g$ if $f(R) \subseteq g(R)$ for all preference profiles $R$. In short, we write $f \subseteq g$ in this case and also say that $g$ is coarser than $f$. An example of an SCF is the Pareto set PO, which selects the alternatives that are Pareto optimal, i.e.,

$$
P O(R)=\left\{x \in A \mid y P_{\mathbf{P a r}} x \text { for no } y \in A\right\}
$$

\footnotetext{
1 Antisymmetry is not required for any of our results to hold. In fact, Theorem 1 is even stronger when also assuming antisymmetric individual preferences (since this only increases the difficulty of constructing a suitable preference profile).

2 Some authors call this strong Pareto optimality. In contrast, an alternative $x$ would be weakly Pareto optimal if there is no alternative $y$ with $y P_{i} x$ for all $i \in N_{R}$. In the case of antisymmetric preferences, the two notions coincide.
} 
An SCF $f$ is called Pareto optimal if it is a refinement of the Pareto set, i.e., if $f \subseteq P O$.

We will restrict attention to so-called majoritarian SCFs, whose outcomes only depend on the majority relation, i.e., to SCFs $f$ such that, for all preference profiles $R, R^{\prime}$,

$$
R_{\mathrm{Maj}}=R_{\mathrm{Maj}}^{\prime} \text { implies } f(R)=f\left(R^{\prime}\right) .
$$

An interesting class of majoritarian SCFs are defined using certain refinements of the majority relation called covering relations. For a given covering relation, the uncovered set contains those alternatives that are not covered by any other alternative. For a comprehensive overview of the theory of covering relations and uncovered sets, see Duggan (2013). A range of varying definitions of the covering relation exist, all of which coincide when restricted to antisymmetric majority relations. We will be concerned with what Duggan refers to as McKelvey covering (Bordes, 1983; McKelvey, 1986).

In order to define the McKelvey covering relation, we need to introduce the notions of strict and weak dominators of a given alternative. The strict dominators $P_{\mathbf{M a j}}(x)$ of an alternative $x \in A$ are defined by the set of all alternatives $y \in A$ that are strictly majority preferred to $x$, i.e., $P_{\mathbf{M a j}}(x):=\left\{y \in A \mid y P_{\mathbf{M a j}} x\right\}$. Analogously, the weak dominators $R_{\mathbf{M a j}}(x)$ of an alternative $x \in A$ are defined as the set of all alternatives $y \in A$ that are weakly majority preferred to $x$, i.e., $R_{\mathbf{M a j}}(x):=\left\{y \in A \mid y R_{\mathbf{M a j}} x\right\}$

Let $C_{R_{\mathrm{Maj}}}$ denote the (McKelvey) covering relation, i.e., for any pair of alternatives $x, y$, the relation $x C_{R_{\text {Maj }}} y$ holds iff each of the following three conditions is satisfied:

(i) $x P_{\text {Maj }} y$

(ii) $P_{\mathrm{Maj}}(x) \subseteq P_{\mathrm{Maj}}(y)$, and

(iii) $R_{\mathrm{Maj}}(x) \subseteq R_{\mathrm{Maj}}(y)$.

As can easily be seen, $C_{R_{\mathrm{Maj}}}$ is transitive and asymmetric. The (McKelvey) uncovered set $U C$ is then defined as

$$
U C(R):=\left\{y \in A \mid x C_{R_{\mathrm{Maj}}} y \text { for no } x \in A\right\} .
$$

Alternative, but equivalent, definitions of the McKelvey uncovered set were used by Dutta and Laslier (1999), Peris and Subiza (1999), and Brandt and Fischer (2008).

It is also well-known (see, e.g., Duggan, 2013) that the McKelvey uncovered set can be characterized as the set of alternatives that dominate every other alternative in at most two steps (of which at most one may be a tie). Formally, the McKelvey uncovered set then consists of all alternatives $x \in A$ such that for all $y \in A \backslash\{x\}$ at least one of the following three conditions is satisfied:

(i) $x R_{\mathrm{Maj}} y$

(ii) there is a $z \in A$ such that $x R_{\text {Maj }} z P_{\text {Maj }} y$, or

(iii) there is a $z \in A$ such that $x P_{\text {Maj }} z R_{\text {Maj }} y$.

For brevity, we will omit any reference to McKelvey in the following and just write "covering" and "uncovered set." 


\section{The Structure of the McKelvey Covering Relation}

In this section we consider the structural properties of the covering relation and observe that any transitive and asymmetric relation can be obtained as the covering relation of some preference profile.

Proposition 1 Let $Q \subseteq A \times A$ be a binary relation. Then the following are equivalent:

(i) $Q$ is transitive and asymmetric.

(ii) There exists a preference profile $R$ such that $C_{R_{\mathrm{Maj}}}=Q$.

Proof The implication from (ii) to $(i)$ is immediate, as for every preference profile $R$ the covering relation $C_{R_{\mathrm{Maj}}}$ is transitive and asymmetric. For the other direction, assume that $Q$ is transitive and asymmetric and let $R$ be a preference profile with $P_{\mathrm{Maj}}=Q$. By virtue of McGarvey's Theorem (McGarvey, 1953) we know such a preference profile exists. It then remains to observe that $(x, y) \in C_{R_{\mathrm{Maj}}}$ iff $(x, y) \in Q$. The "if"-direction is immediate. For the "only if"-direction, consider an arbitrary edge $(x, y) \in Q$. By construction of $R$, also $x P_{\text {Maj }} y$ and, by transitivity of $Q=P_{\text {Maj }}$, we obtain $P_{\text {Maj }}(x) \subseteq P_{\text {Maj }}(y)$. To see that also $R_{\mathrm{Maj}}(x) \subseteq R_{\mathrm{Maj}}(y)$, consider an arbitrary $z \in R_{M}(x)$ and assume for contradiction that $y P_{\mathbf{M a j}} z$. Again by transitivity of $P_{\mathbf{M a j}}$, then $x P_{\mathrm{Maj}} z$, a contradiction. It follows that $z \in R_{\mathrm{Maj}}(y)$.

Interestingly, the implication from $(i)$ to $(i i)$ does not hold if $R_{\mathrm{Maj}}$ is required to be antisymmetric (e.g., when the number of agents is odd). We are not aware of a non-trivial characterization of potential covering relations for this case. ${ }^{3}$

A result analogous to Proposition 1 was shown by Dushnik and Miller (1941) for the Pareto relation. They proved that for any transitive and asymmetric relation $Q \subseteq A \times A$ there exists a preference profile $R$ such that $P_{\text {Par }}=Q$. Note that this does not imply Proposition 1 as the majority relation outside $Q$ may be very different in the preference profile $R$ instantiating $Q$ as the Pareto relation, and a preference profile $R^{\prime}$ instantiating $Q$ as the covering relation. In our proof, in particular, all edges outside $Q$ are required to be majority ties.

Regarding the internal structure of the uncovered set, it was already shown by Moulin (1986) that any complete binary relation without a (non-trivial) Condorcet winner is the majority relation between uncovered alternatives for some preference profile. While Moulin (1986) proved this result for tournaments only, the argument can easily be adapted to cover our setting, in which majority ties are allowed.

3 Consider, for instance, the simple case of $A=\{a, b, c\}$ and $Q=\{(a, b),(a, c)\}$, which is easily seen not to be the covering relation for any preference profile. 


\section{The McKelvey Uncovered Set and the Pareto Set are Majority-equivalent}

In this section, we consider the relationship between the uncovered set and the Pareto set for profiles that yield the same majority relation (i.e., consistent preference profiles).

Our main result shows that for every preference profile $R$, we can find another preference profile $R^{\prime}$ consistent with $R_{\text {Maj }}$ such that the uncovered set of $R$ and the Pareto set of $R^{\prime}$ coincide. This has a number of consequences. First, if we assume that only the majority relation is known, the uncovered set not only coincides with the Pareto optimal alternatives for some consistent preference profile, but also consists of precisely those alternatives that are Pareto optimal for every consistent preference profile. Moreover, there exists a consistent profile in which all covered alternatives are Pareto dominated. Secondly, the theorem implies that the uncovered set can be characterized as the coarsest majoritarian Pareto optimal SCF.

Theorem 1 For every preference profile $R$, there is another preference profile $R^{\prime}$ with $R_{\mathbf{M a j}}=R_{\mathbf{M a j}}^{\prime}$ such that

$$
U C(R)=P O\left(R^{\prime}\right)
$$

Proof Consider an arbitrary preference profile $R$. Then, for every alternative $y \notin U C(R)$ there is some $x \in U C(R)$ such that $x C_{R_{\text {Maj }}} y$. Thus, let $U C(R)=\left\{x_{1}, \ldots, x_{\ell}\right\}$ and associate with every $x_{i} \in U C(R)$ a (possibly empty) set $f\left(x_{k}\right) \subseteq\left\{x^{\prime} \in A \mid x C_{R_{\mathrm{Maj}}} x^{\prime}\right\}$ such that

$$
f\left(x_{k}\right) \cap f\left(x_{k^{\prime}}\right)=\emptyset \text { for } 1 \leq k<k^{\prime} \leq \ell \text { and } f\left(x_{1}\right) \cup \cdots \cup f\left(x_{\ell}\right)=A \backslash U C(R) .
$$

Define the relation $F \subseteq C_{R_{\mathrm{Maj}}}$ such that, for all alternatives $x$ and $y$,

$$
x F y \quad \text { iff } \quad x \in U C(R) \text { and } y \in f(x) .
$$

We construct a preference profile $R^{\prime}$ such that $R_{\mathrm{Maj}}^{\prime}=R_{\mathrm{Maj}}$ and $R_{\mathrm{Par}}^{\prime}=F$. It can readily be appreciated that then $U C(R)=P O\left(R^{\prime}\right)$, as desired.

For notational convenience we denote $f\left(x_{k}\right)$ by $X_{k}$. For any subset $Y$ of alternatives, by $\vec{Y}$ and $\stackrel{\leftarrow}{Y}$ we denote an enumeration of $Y$ and its inverse, respectively, i.e., if $\vec{Y}=y_{1}, \ldots, y_{k}$ then $\overleftarrow{Y}=y_{k}, \ldots, y_{1}$.

First, we generate the relation $F$. To this end, we introduce two agents, $i_{F}$ and $j_{F}$ with preferences given by the following two sequences:

$$
\begin{gathered}
i_{F}: x_{1}, \vec{X}_{1}, \ldots, x_{\ell}, \vec{X}_{\ell} \\
j_{F}: x_{\ell}, \overleftarrow{X}_{\ell}, \ldots, x_{1}, \overleftarrow{X}_{1}
\end{gathered}
$$

Letting $R^{F}=\left(R_{i_{F}}, R_{j_{F}}\right)$, we thus have $R_{\text {Par }}^{F}=F$.

Furthermore, for every pair $(v, w)$ in $R_{\text {Maj }}$ that is not contained in $F$ we also introduce two additional agents $i_{v w}$ and $j_{v w}$. We distinguish five cases and denote by $R^{v w}$ the profile $\left(R_{i_{v w}}, R_{j_{v w}}\right)$. Without loss of generality and for notational convenience, we will usually assume that $v^{+}=x_{1}$ and $w^{+}=x_{2}$. 
Case 1 There is a $v^{+} \in U C(R)$ such that $v, w \in f\left(v^{+}\right)$. Let $V=f\left(v^{+}\right) \backslash$ $\{v, w\}$. For an illustration see Figure 1a. Then, define the preferences of $i_{v w}$ and $j_{v w}$ by the following lists:

$$
\begin{aligned}
& i_{v w}: v^{+}, v, w, \vec{V}, x_{2}, \vec{X}_{2}, \ldots, x_{\ell}, \vec{X}_{\ell} \\
& j_{v w}: x_{\ell}, \overleftarrow{X}_{\ell}, \ldots, x_{2}, \overleftarrow{X}_{2}, v^{+}, \overleftarrow{V}, v, w
\end{aligned}
$$

Observe that in this case, we have $P_{\mathbf{M a j}}^{v w}=F \cup\{(v, w)\}$.

Case 2 There are $v^{+}, w^{+} \in U C(R)$ with $v \in f\left(v^{+}\right)$and $w \in f\left(w^{+}\right)$. Let $V=f\left(v^{+}\right) \backslash\{v\}$ and $W=f\left(w^{+}\right) \backslash\{w\}$. Observe that in this case we have that $v^{+} C_{R_{\mathrm{Maj}}} v$ for $R$ and, hence, also $v^{+} P_{\mathrm{Maj}} w$. For an illustration see Figure 1b. In this case define the preferences of $i_{v w}$ and $j_{v w}$ as

$$
\begin{aligned}
& i_{v w}: v^{+}, \vec{V}, v, w^{+}, w, \vec{W}, x_{3}, \vec{X}_{3}, \ldots, x_{\ell}, \vec{X}_{\ell} \\
& j_{v w}: x_{\ell}, \overleftarrow{X}_{\ell}, \ldots, x_{3}, \overleftarrow{X}_{3}, w^{+}, \overleftarrow{W}, v^{+}, v, w, \overleftarrow{V}
\end{aligned}
$$

It thus follows that $P_{\mathrm{Maj}}^{v w}=F \cup\left\{(v, w),\left(v^{+}, w\right)\right\}$.

Case 3 There are $v^{+}, w^{+} \in U C(R)$ with $v=v^{+}$and $w=w^{+}$. Let $V=$ $f\left(v^{+}\right)$and $W=f\left(w^{+}\right)$. Observe that in this case we have for $R$ that $w^{+} C_{R_{\mathrm{Maj}}} x$ and, hence, also $v^{+} P_{\text {Maj }} x$ for all $x \in W$. The situation is depicted in Figure 1c. Now define

$$
\begin{aligned}
& i_{v w}: v^{+}, \vec{V}, w^{+}, \vec{W}, x_{3}, \vec{X}_{3}, \ldots, x_{\ell}, \vec{X}_{\ell} \\
& j_{v w}: x_{\ell}, \overleftarrow{X}_{\ell}, \ldots, x_{3}, \overleftarrow{X}_{3}, v^{+}, w^{+}, \overleftarrow{W}, \overleftarrow{V}
\end{aligned}
$$

Observe that now $P_{\mathbf{M a j}}^{v w}=F \cup\left\{\left(v^{+}, w^{+}\right)\right\} \cup\left\{\left(v^{+}, x\right) \mid x \in W\right\}$.

Case 4 There are $v^{+}, w^{+} \in U C(R)$ with $v=v^{+}$and $w \in f\left(w^{+}\right)$. Let $V=$ $f\left(v^{+}\right)$and $W=f\left(w^{+}\right) \backslash\{w\}$. The situation is depicted in Figure 1d. In this case define the preferences of $i_{v w}$ and $j_{v w}$ as follows:

$$
\begin{aligned}
& i_{v w}: v^{+}, \vec{V}, w^{+}, w, \vec{W}, x_{3}, \vec{X}_{3}, \ldots, x_{\ell}, \vec{X}_{\ell}, \\
& j_{v w}: x_{\ell}, \overleftarrow{X}_{\ell}, \ldots, x_{3}, \overleftarrow{X}_{3}, w^{+}, \overleftarrow{W}, v^{+}, w, \overleftarrow{V}
\end{aligned}
$$

Accordingly, we also have $P_{\mathbf{M a j}}^{v w}=F \cup\left\{\left(v^{+}, w\right)\right\}$.

Case 5 There are $v^{+}, w^{+} \in U C(R)$ with $v \in f\left(v^{+}\right)$and $w=w^{+}$. Let $V=$ $f\left(v^{+}\right) \backslash\{v\}$ and $W=f\left(w^{+}\right)$. Observe that in this case we have for $R$ that $v^{+} C_{R_{\mathrm{Maj}}} v$ and $w^{+} C_{R_{\mathrm{Maj}}} x$ for all $x \in W$. As a consequence, also $v^{+} P_{\mathbf{M a j}} w^{+}$, and thus $v^{+} P_{\mathbf{M a j}} x$ and $v P_{\mathbf{M a j}} x$ for all $x \in W$. The situation is depicted in Figure 1e. In this case define:

$$
\begin{aligned}
& i_{v w}: v^{+}, \overleftrightarrow{V}, v, w^{+}, \vec{W}, x_{3}, \vec{X}_{3}, \ldots, x_{\ell}, \vec{X}_{\ell} \\
& j_{v w}: x_{\ell}, \overleftarrow{X}_{\ell}, \ldots, x_{3}, \overleftarrow{\leftarrow}_{3}, v^{+}, v, w^{+}, \overleftarrow{W}, \overleftarrow{V}
\end{aligned}
$$

Hence, $P_{\mathbf{M a j}}^{v w}=F \cup\left(\left\{v^{+}, v\right\} \times\left(W \cup\left\{w^{+}\right\}\right)\right)$. 


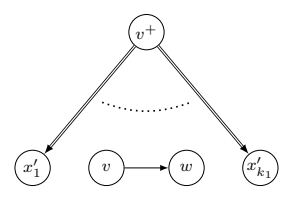

(a) Case 1

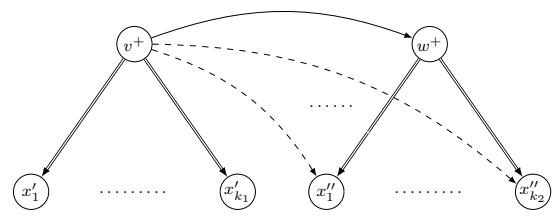

(c) Case 3

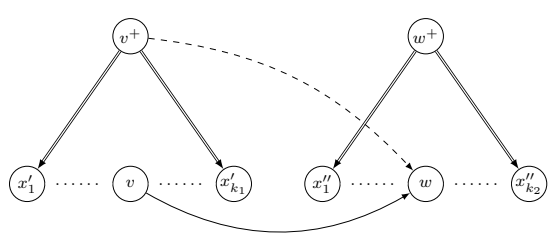

(b) Case 2

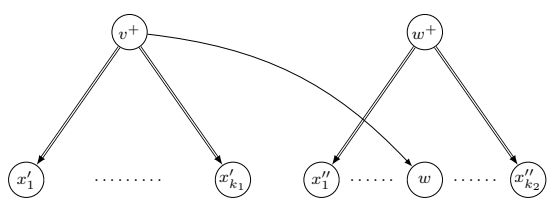

(d) Case 4

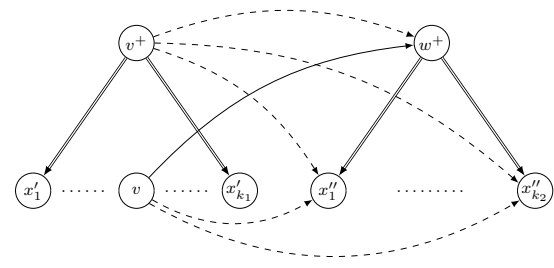

(e) Case 5

Fig. 1: The five cases distinguished in the proof of Theorem 1. The relation $F$ is indicated by double arrows.

Let $R_{\mathrm{Maj}} \backslash F$ be given by $\left\{\left(v_{1}, w_{1}\right), \ldots,\left(v_{p}, w_{p}\right)\right\}$ and consider the profile

$$
R^{\prime}=\left(R_{1}, \ldots, R_{2 p+2}\right)=\left(R_{i_{F}}, R_{j_{F}}, R_{i_{v_{1} w_{1}}}, \ldots, R_{i_{v_{p} w_{p}}}\right) .
$$

Some reflection reveals that $R_{\mathbf{M a j}}^{\prime}=R_{\mathbf{M a j}}$. Also observe that $F \subseteq R_{i}$ for all $1 \leq i \leq 2 p+2$. Moreover, $x P_{i_{F}} y$ iff $y P_{j_{F}} x$, for all $(x, y) \notin F$. Hence, $R_{\text {Par }}^{\prime}=F$. Since, $x \notin U C(R)$ iff $y F x$ for some $y \in U C(R)$, it follows that $P O\left(R^{\prime}\right)=U C(R)$, which concludes the proof.

For the next corollary we additionally need the (easy-to-prove) fact that the uncovered set $U C$ is Pareto optimal, which to the best of our knowledge was first mentioned by Bordes (1983). We formalize it in the following lemma for the sake of completeness. Note that this result relies on the definition of the majority relation via simple majority rule (see, e.g., Gaertner, 2009, p. 39). If 
majorities are defined via (the less common) absolute majority rule, then the uncovered set only satisfies weak Pareto optimality (Duggan, 2013).

Lemma 1 (Bordes, 1983) The Pareto relation is a subrelation of the $M c K$ Kelvey covering relation. Hence, $U C \subseteq P O$.

Proof Let $R$ be a preference profile and $x, y \in A$ alternatives such that $x P_{\text {Par }} y$. To show that then also $x C_{R_{\text {Maj }}} y$, first suppose $z P_{\text {Maj }} x$ for some alternative $z \in A$. Since individual preferences are assumed to be transitive it follows that $z P_{\mathbf{M a j}} y$. The case of $z R_{\mathbf{M a j}} x$ (implying $z R_{\mathbf{M a j}} y$ ) is analogous and $x P_{\mathbf{M a j}} y$ is an immediate consequence of $x P_{\mathbf{P a r}} y$.

The following corollary, which follows from Theorem 1 and Lemma 1, provides a characterization of the McKelvey uncovered set via Pareto optimality. ${ }^{4}$

Corollary 1 A majoritarian SCF $f$ is Pareto optimal iff $f \subseteq U C$. Consequently, the McKelvey uncovered set is the coarsest Pareto optimal majoritarian $S C F$.

Proof Lemma 1 establishes that the McKelvey uncovered set $U C$ (and any refinement of it) is Pareto optimal. To prove the other direction by contraposition, consider an arbitrary majoritarian SCF $f$ such that $f \not \subset U C$. Then, there is a profile $R$ and an alternative $a$ such that $a \in f(R)$ and $a \notin U C(R)$. By Theorem 1, some preference profile $R^{\prime}$ exists such that $R_{\mathrm{Maj}}=R_{\mathrm{Maj}}^{\prime}$ and $U C(R)=P O\left(R^{\prime}\right)$. Having assumed $f$ to be majoritarian, $f\left(R^{\prime}\right)=f(R)$ and hence $a \in f\left(R^{\prime}\right)$. It also follows that $a \notin P O\left(R^{\prime}\right)$ and we may conclude that $f$ is not Pareto optimal.

\section{Potential Extensions}

We consider two natural extensions of Theorem 1 and show that neither of them holds.

\subsection{Constant number of agents}

The proof of Theorem 1 crucially depends on the assumption of a variable electorate since $R^{\prime}$ usually has a different number of agents than $R$. The fact that the same result cannot be achieved with a constant number of agents is exhibited by the following minimal, computer-generated example. ${ }^{5}$ The majority relation $R_{\text {Maj }}$ depicted in Figure 2 contains a unique covering edge $a C_{R_{\mathrm{Maj}}} b$ and can be realized by 3 agents. ${ }^{6}$

\footnotetext{
${ }^{4}$ Corollary 1 also entails an analogous weaker result for the special case of tournaments (i.e., antisymmetric majority relations $R_{\mathrm{Maj}}$ ), which was used as a Lemma by Brandt and Geist (2014).

5 The whole example was obtained from and proved minimal by an automated computer search based on the method developed by Brandt et al. (2014).

${ }^{6}$ In fact, any tournament of size 7 can be realized by 3 agents.
} 


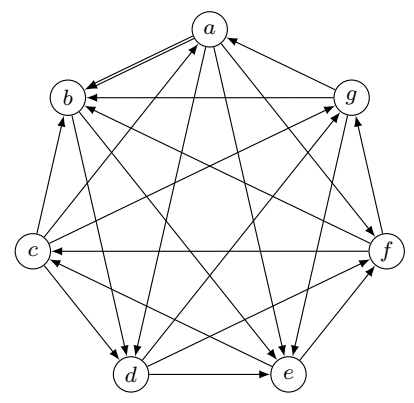

(a) majority relation $R_{\mathrm{Maj}}$

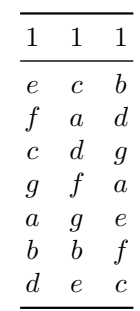

(b) a consistent preference profile $R$

Fig. 2: A minimal example of a majority relation $R_{\mathbf{M a j}}$ for which the transition from $b \notin U C(R)$ to $b \notin P O(R)$ increases the minimal number of agents required to realize this relation.

If we, however, require $b$ to be Pareto dominated, i.e., $x P_{\mathbf{P a r}} b$ for $a t$ least one of the alternatives $x P_{\mathbf{M a j}} b$, then the minimal number of agents required to realize $R_{\text {Maj }}$ (together with the additional requirement of $x P_{\mathbf{P a r}} b$ ) rises to 5 , which has been verified on a computer. ${ }^{7}$

This example also shows that Corollary 1 does not in general hold for all constant electorates. To see this, let the number of agents be fixed at three and define an SCF $f$ such that for all preference profiles $R^{\prime}$,

$$
f\left(R^{\prime}\right)= \begin{cases}A & \text { if } R^{\prime} \text { is consistent with } R_{\mathbf{M a j}} \\ U C\left(R^{\prime}\right) & \text { otherwise }\end{cases}
$$

where $R_{\text {Maj }}$ is the majority relation in Figure 2. Thus, $f$ is a coarsening of $U C$ but, due to the electorate being fixed at three agents, $f$ is still Pareto optimal.

4.2 Equivalence of the covering relation and the Pareto relation

With Theorem 1 in mind, it is a natural question whether, given a preference profile $R$, one can even obtain a consistent preference profile $R^{\prime}$ (i.e., $R_{\mathrm{Maj}}=$ $R_{\mathrm{Maj}}^{\prime}$ ) in which the Pareto relation coincides with the covering relation (i.e., $\left.P_{\text {Par }}^{\prime}=C_{R_{\mathrm{Maj}}}\right)$. Unfortunately, the answer to this question is negative as the following counterexample shows.

Consider the preference profile $R$ and the corresponding majority relation $R_{\mathrm{Maj}}$ in Figure 3 and note that all strict majority edges are also covering edges, i.e., $P_{\mathrm{Maj}}=C_{R_{\mathrm{Maj}}}$. Therefore, constructing a consistent preference profile $R^{\prime}$ with $P_{\mathbf{P a r}}^{\prime}=C_{R_{\mathrm{Maj}}}$, in this particular example, means to construct

\footnotetext{
7 This even holds when individual preferences are allowed to be weak orders.
} 


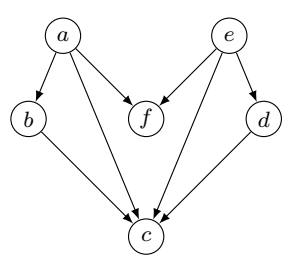

(a) majority relation $R_{\mathrm{Maj}}$, whose strict part $P_{\text {Maj }}$ coincides with the covering relation $C_{R_{\mathrm{Maj}}}$

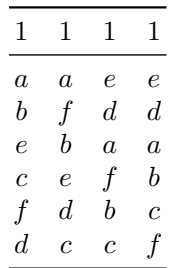

(b) a consistent preference profile $R$

Fig. 3: An example of a majority relation $R_{\mathrm{Maj}}$ whose covering relation $C_{R_{\mathrm{Maj}}}$ cannot be obtained as the Pareto relation of a consistent preference profile.

a consistent preference profile $R^{\prime}$ such that $P_{\text {Par }}^{\prime}=C_{R_{\mathrm{Maj}}}=P_{\mathrm{Maj}}$. We now show - using computer-aided solving techniques - that such a profile does not exist.

For $P_{\text {Par }}^{\prime}=P_{\mathbf{M a j}}$ to hold, $R^{\prime}$ may only contain agents with individual preferences $R_{i}^{\prime}$ that respect all given strict majority edges as Pareto edges, i.e., for which $P_{i}^{\prime} \supseteq P_{\text {Maj }}$. This will stand in conflict with being able to maintain the majority ties in $R_{\mathbf{M a j}}^{\prime}=R_{\mathbf{M a j}}$. We used an ASP program (Answer Set Programming, a declarative problem-solving paradigm; see, e.g., Gebser et al. (2011, 2012); in our case the packaged grounder/solver CLASP) to compute all 22 candidates $R_{i}^{\prime \prime}$ for complete, antisymmetric, and transitive preference relations such that $P_{i}^{\prime \prime} \supseteq P_{\mathbf{M a j}}$. (One such ordering is $e, d, a, f, b, c$.) We would now have to construct a preference profile using only these 22 candidates for individual preference relations as building blocks. A small IP (Integer Program, see, e.g., Schrijver (1986); the performance of the open-source solver LPSOLVE satisfied our requirements) suffices to show that this is not possible: it contains 22 integer-valued variables $x_{R_{i}^{\prime \prime}}$, which denote, for each of the 22 preference relations, how many agents with this particular preference relation are contained in $R^{\prime}$. Furthermore, it contains one constraint per indifference edge in the majority relation (of which there are 7, namely $(a, d),(a, e),(b, d),(b, e)$, $(b, f),(c, f)$, and $(d, f))$. Each of these constraints postulates for one indifference edge $(y, z)$ that $0=\sum_{R_{i}^{\prime \prime}} s\left((y, z), R_{i}^{\prime \prime}\right) \cdot x_{R_{i}^{\prime \prime}}$, where $s\left((y, z), R_{i}^{\prime \prime}\right)=1$ if $(y, z) \in R_{i}^{\prime \prime}$, and $s\left((y, z), R_{i}^{\prime \prime}\right)=-1$ otherwise. With the additional constraints that $x_{R_{i}^{\prime \prime}} \geq 0$ for all $R_{i}^{\prime \prime}$ and $\sum_{R_{i}^{\prime \prime}} x_{R_{i}^{\prime \prime}} \geq 1$ the IP solver returns that there are no feasible solutions to this problem, which completes the proof. $^{8}$

8 The same counterexample also applies to the case of weak individual orders (i.e., without the assumption of antisymmetry of $R_{i}$ ). In this case there are 256 instead of 22 candidates for individual preferences relations, and additional constraints for the Pareto edges are 


\section{References}

G. Bordes. On the possibility of reasonable consistent majoritarian choice: Some positive results. Journal of Economic Theory, 31(1):122-132, 1983.

F. Brandt and F. Fischer. Computing the minimal covering set. Mathematical Social Sciences, 56(2):254-268, 2008.

F. Brandt and C. Geist. Finding strategyproof social choice functions via SAT solving. In Proceedings of the 13th International Conference on Autonomous Agents and Multi-Agent Systems (AAMAS), pages 1193-1200. IFAAMAS, 2014.

F. Brandt, C. Geist, and H. G. Seedig. Identifying $k$-majority digraphs via SAT solving. In Proceedings of the 1st AAMAS Workshop on Exploring Beyond the Worst Case in Computational Social Choice (EXPLORE), 2014.

J. Duggan. Uncovered sets. Social Choice and Welfare, 41(3):489-535, 2013.

B. Dushnik and E. W. Miller. Partially ordered sets. American Journal of Mathematics, 63(3):600-610, 1941.

B. Dutta and J.-F. Laslier. Comparison functions and choice correspondences. Social Choice and Welfare, 16(4):513-532, 1999.

W. Gaertner. A Primer in Social Choice Theory: Revised Edition. LSE Perspectives in Economic Analysis. Oxford University Press, 2009.

M. Gebser, R. Kaminski, B. Kaufmann, M. Ostrowski, T. Schaub, and M. Schneider. Potassco: The Potsdam answer set solving collection. AI Communications, 24(2):107-124, 2011.

M. Gebser, R. Kaminski, B. Kaufmann, and T. Schaub. Answer set solving in practice. Synthesis Lectures on Artificial Intelligence and Machine Learning, 6(3):1-238, 2012.

D. C. McGarvey. A theorem on the construction of voting paradoxes. Econometrica, 21(4):608-610, 1953.

R. D. McKelvey. Covering, dominance, and institution-free properties of social choice. American Journal of Political Science, 30(2):283-314, 1986.

H. Moulin. Choosing from a tournament. Social Choice and Welfare, 3(4): 271-291, 1986.

J. E. Peris and B. Subiza. Condorcet choice correspondences for weak tournaments. Social Choice and Welfare, 16(2):217-231, 1999.

A. Schrijver. Theory of Linear and Integer Programming. John Wiley \& Sons, 1986.

required, which makes the resulting IP significantly larger, but still solvable within less than one second. 